

\title{
Introduction
}

Retinitis pigmentosa (RP) is a term often used to refer to all inherited diseases that affect the photoreceptor cells of the retina. However, the use of this singular term belies the fact that thousands of different genetic variations distributed across dozens if not hundreds of different genes are capable of causing the dysfunction and/or premature death of this specialized class of neurons. Although all forms of inherited photoreceptor disease combined affect fewer than one in 3000 individuals in the general population, this class of diseases is worthy of general scientific and medical interest for a number of reasons. First, vision is an extremely important aspect of every person's health and the photoreceptor cell is an absolutely indispensable component of the visual system. Second, the retina is in many ways a microcosm of the entire body, containing many gene products and cell types that are important to other important organ systems, including the kidney, cochlea, and brain. Thus, the elucidation of a given physiologic or pathophysiologic process in the retina will often have a very meaningful correlation elsewhere in the body. Third, compared to other organs, the retina is very accessible to direct observation and therapeutic intervention. The combination of slit lamp biomicroscopy and the newest implementations of optical coherence tomography [1] allow an ophthalmologist to examine the retina of living patients with a resolution approaching that of light microscopy. The thin structure of the retina

Publisher's Disclaimer: This is a PDF file of an unedited manuscript that has been accepted for publication. As a service to our customers we are providing this early version of the manuscript. The manuscript will undergo copyediting, typesetting, and review of the resulting proof before it is published in its final citable form. Please note that during the production process errors may be discovered which could affect the content, and all legal disclaimers that apply to the journal pertain. 
allows its function and dysfunction to be assessed using instruments (e.g., perimeters) capable of mapping measurements to specific subregions. Drugs of all types can be injected into the vitreous cavity and even the subretinal space can be accessed safely by most retinal surgeons. Fourth, the very orderly arrangement of the neurons of the retina coupled with the excitability of the photoreceptors by visible light allows the many neuronal pathways of the retina to be assessed more easily and more accurately than deeper and more complex portions of the central nervous system. Finally, the extensive genetic, mechanistic, and phenotypic heterogeneity of inherited diseases of the retina make it an ideal model system for developing efficient strategies for identifying the most meaningful relationships among phenotype, genotype, disease nomenclature and disease mechanism.

To expand the latter point, variations in numerous genes [2] are known to result in the constellation of clinical findings that most ophthalmologists would call "retinitis pigmentosa" (figure 1). The fact that variations in multiple genes can cause a single phenotype is well known and is frequently referred to using the phrase "genetic heterogeneity of the disease". However, analogous heterogeneity can also occur in the phenotype dimension [3,4]. That is, some variations in the $A B C A 4$ and $R D S$ genes cause a phenotype very similar to that of the patient illustrated in figure 1 while other variations in these same genes cause macula-selective phenotypes (Stargardt disease and pattern dystrophy) that are considered to be "different diseases" by clinicians. Although confusing for patients, clinicians and laboratory scientists, there is nothing scientifically "wrong" with the lack of a one to one relationship between genotypes, phenotypes and historical disease names. In fact, it would be amazing if nineteenth century clinicians had devised a nomenclature that agreed perfectly with twenty-first century molecular knowledge.

Thus, "genetic heterogeneity" of a given "disease" is simply a way of stating that current clinical knowledge, nomenclature, diagnostic instruments, or examination methods are insufficiently refined to reliably identify and separate different genetic causes of a certain phenotype. The improvement of the genotype-phenotype-mechanism-nomenclature map can and will occur in at least two directions. One can investigate and identify the phenotypic range of genetic variations in a specific gene and one can identify the genotypic range of a group of patients who share certain clinical features. At any given point in the evolution of clinical or molecular knowledge, one investigative approach will be more fruitful than the other. However, by the time the genotype-phenotype landscape is fully understood, both routes will have been extensively utilized.

\section{Genetic Testing}

There are at least six classes of therapy that have been explored to a meaningful degree for treatment of the various mechanistic types and various stages of human photoreceptor degenerations (Table 1) and there are three reasons that genetic testing should be the first entry on this list. First, as all those who care for individuals affected with these disorders will attest, a firm and specific diagnosis is a great comfort to patients and their families [5]. A reliable molecular diagnosis allows accurate genetic counseling to be provided, and as more and more genotypephenotype correlation data are gathered, the molecular diagnosis also allows a more accurate prognosis to be given in many cases. Second, a fairly precise 
knowledge of the mechanism and stage of disease is always desirable before choosing any therapy for a specific patient. That is, providing an unregulated copy of a normal gene might not be expected to be very helpful for a disease caused by a toxic effect of a mutant allele while the very same treatment might work well for a recessive disease in a patient with no functional copy of the gene. Similarly, gene replacement therapy targeting photoreceptor cells would not be expected to be of much benefit for the late stages of a disease in which most of the photoreceptor cells have already been lost but might work very well if administered very early in the disease while most of the photoreceptor cells are still healthy [6]. Third, full deployment of low-cost, high throughput genetic testing methods throughout large populations will be necessary to identify the ideal patient populations for enrollment in clinical trials of new iterations of all of the other classes of therapy.

In the past few years, a variety of new molecular methods have become available that allow large numbers of genes to be assayed in large numbers of patients at fairly low cost [7, 8]. In parallel, new strategies have been developed for combining these methods to take maximal advantage of their strengths and to minimize their weaknesses [8]. Another advance has been the development of algorithmic approaches for distinguishing true disease-causing mutations from non-disease-causing polymorphisms [9].

\section{Environmental Modification}

Nutritional supplementation - The fact that deficiencies of certain vitamins can cause abnormalities of vision - occasionally quite profound - has caused some investigators to imagine that supplementation of the same or related substances might prevent or delay the photoreceptor cell death associated with some inherited retinal diseases. Functionally, this class of treatment overlaps the "small molecule drugs" in the next section. However, molecules that can be increased or reduced by altering one's diet do differ in many practical and legal ways from molecules that are manufactured by a pharmaceutical company. In 1993, Berson, et al., reported a small but statistically significant preservation of the electroretinogram for adult retinitis pigmentosa patients who took 15,000 units of vitamin A palmitate per day for five years [10]. At the time this study was performed, it was not feasible to subdivide patients according to the specific genetic causes of their disease. An additional study in a mouse model of rhodopsin associated RP showed that vitamin A supplementation reduced the rate of loss of the ERG in that model [11]. In contrast to these favorable reports, a more recent study suggests that vitamin A supplementation may actually be harmful for photoreceptor diseases associated with ABCA4 dysfunction [12]. This is especially concerning given that the nutritional supplements that are widely used for agerelated macular degeneration contain high doses of beta carotene, a vitamin A precursor [13]. Indiscriminate use of these supplements for "juvenile macular degeneration" (e.g., Stargardt disease) could cause harm. Now that animal models exist for numerous genetic subtypes of photoreceptor disease, and human genetic testing is becoming more widely available, it seems wise to limit high dose vitamin supplementation to specific genetic subtypes of inherited eye disease that have been shown, at least in animals, to benefit from the treatment. 
Avoidance of light - Three lines of evidence suggest that reduction of some types of light exposure could be a beneficial intervention for some patients with inherited photoreceptor degenerations. First, chronic exposure of normal rodents to even modest amounts of visible light will cause a severe photoreceptor degeneration [14]. Second, Jacobson and co-workers observed a worsening of rhodopsin associated retinitis pigmentosa in dogs when their eyes were exposed to repeated bright flashes of light associated with retinal photography [15]. Third, Travis and co-workers showed that abnormal lipofuscin accumulation in Abca4 deficient mice is exacerbated by light and does not occur at all if the animals are reared in total darkness [16]. Although there are undoubtedly large species differences with respect to the levels and duration of light exposure that are toxic and one must be cautious in directly translating murine results to humans, it seems reasonable for all patients with inherited photoreceptor degenerations - especially those with ABCA4 and rhodopsin-associated disease - to avoid unprotected exposure to prolonged bright light pending further investigation in animal models and human clinical trials.

\section{Drugs}

Small molecule - Given that the machinery of vision involves ion channels, signaling cascades, and synaptic connections that are similar to pharmaceutically accessible targets in other organ systems, it seems reasonable to imagine that some targets can be identified in this system that will be reachable with small molecule agents (i.e., less than 1000 daltons in size) that can be delivered through the bloodstream, through intravitreal injection, or via topical administration to the eye. The following three examples of such interventions are representative of the promise of this idea. First, the recognition of the light dependent exchange of vitamin A derivatives between the photoreceptors and the retinal pigment epithelium as a key factor in the pathogenesis of $A B C A 4$ - mediated retinal disease caused investigators to consider the therapeutic potential of small molecule drugs that were known to interfere with this cycle. Radu and coworkers showed that chronic intraperitoneal injection of fenretinide to abca4 ${ }^{-/}$mice arrested the accumulation of the toxic fluorophore $\mathrm{A} 2 \mathrm{E}$ in the retinal pigment epithelium of these animals [17]. Another example of a small molecule intervention is based upon the hypothesis that oxygen plays a key role in the photoreceptor death of retinitis pigmentosa. Photoreceptor cells consume more oxygen per gram than any other cell type [18], and when they die, the resting oxygen tension in the retina rises considerably. Komeima and coworkers hypothesized that the elevated oxygen tension in the retina that results from the death of rod photoreceptors is in turn toxic to the remaining cone photoreceptors [19]. They systemically administered a mixture of four antioxidant drugs to a mouse model of retinitis pigmentosa $(r d l)$ and observed a twofold increase in cone cell density compared to untreated animals. The third example of a small molecule intervention is a neuroprotective agent known as tauroursodeoxycholic acid (TUDCA). Phillips and coworkers showed that chronic systemic administration of this agent dramatically slowed the loss of photoreceptors in a mouse model of RP ( $r d 10)$ [20].

Large molecule - It is well known that certain growth factors can delay or prevent the apoptotic death of photoreceptors in several different forms of inherited photoreceptor degeneration [21]. This is particularly exciting because such a treatment might prove to be effective for several mechanistic subtypes of human disease and thus might be usable in 
situations where the molecular cause of the disease cannot be clearly established. Although the precise mechanism through which growth factors exert their beneficial effects are still being worked out, recent studies suggest that the prevention of apoptosis is associated in some cases (e.g., CNTF) with retinal remodeling and a dedifferentiation of the photoreceptors [22]. Thus, it may turn out that as with many very powerful therapeutic agents, controlling the dose of the drug within a fairly narrow window will be necessary for a beneficial effect for patients with photoreceptor disease. Alternatively, these agents may be used as a temporizing means to keep the photoreceptors alive long enough for more durable cures to be delivered.

\section{Gene Therapy}

Given that inherited photoreceptor degenerations are caused at the most basic level by variations in genomic DNA, it is not surprising that many forms of treatment that are envisioned or actually under investigation seek to employ the mechanisms of gene expression to effect a therapeutic benefit. These interventions can be grouped into three classes: gene replacement, mutant allele suppression and therapeutic protein delivery. The most common method for introducing a transgene into the retina is a viral vector although some nonviral methods such as polylysine nanoparticles [23] have recently shown promise in the retina.

The majority of human photoreceptor diseases are inherited in an autosomal recessive manner and in most of these the mechanism of disease is traceable to the lack of a gene product rather than the presence of a toxic allele. One of the most dramatic examples of this is a form of childhood blindness caused by the lack of a retinoid isomerase encoded by a gene (RPE65) expressed in the retinal pigment epithelium (RPE). There are four features of this disease that made it an ideal early target for a clinical trial of human gene replacement therapy: 1) the retina is normally formed in affected patients and the blindness (at least initially) results solely from the lack of an enzymatic activity in the RPE; 2) the phenotype is so severe that even a modest improvement in function is readily detectable; 3 ) the dysfunctional gene is small enough to fit into an adeno-associated viral vector; 4) a naturally occurring canine model exists, enabling proof of concept testing to be performed in a large eyed mammal. In 2001, Acland and co-workers reported the efficacy of gene replacement therapy in the canine model [24] and seven years later, three different groups reported promising results following treatment of human patients with adeno-associated-virusmediated gene replacement therapy [25-27].

Approximately $15 \%$ of human photoreceptor disease is inherited in an autosomal dominant fashion and in many of these cases the mechanism of disease likely involves a toxic effect of the mutant allele. Various strategies ranging from ribozymes to small inhibitory RNAs have been used with varying success to selectively suppress the mutant allele. A recent example of the therapeutic success of such a treatment in an animal model of a human photoreceptor disease was a study by Gorbatyuk, et al., in which an adeno-associated viral vector bearing an allele-independent ribozyme was used to partially rescue the RP phenotype in a murine model of rhodopsin-associated retinitis pigmentosa [28]. 
A third strategy for using gene transfer to mitigate photoreceptor disease is to use it as a means to steadily deliver a "large molecule drug" such as a growth factor. For example, Buch, et al., succeeded in ameliorating the disease phenotype in two murine models of RP by using an adeno-associated virus to deliver a transgene encoding glial cell line-derived neurotrophic factor [29].

With all three of these gene transfer strategies it will likely prove valuable if not essential to be able to fine tune the level of transgene expression after the gene transfer is accomplished. This can be done by incorporating a drug sensitive promoter in the therapeutic construct. For example, in primates, Stieger and coworkers recently demonstrated effective control of a transgene for years with oral doxycycline [30].

\section{Cell Therapy}

For many human photoreceptor diseases, a large fraction of the photoreceptor cells have already been lost at the time the diagnosis is first made. In these situations, it would be ideal to be able to replace these cells with either an autologous or heterologous cell transplant. Deriving the therapeutic cells from the patients themselves would have the advantage of a perfect immunologic match, but the disadvantage that the genetic abnormality responsible for the disease would need to be treated as part of the process of preparing the cells for transplant. Obtaining therapeutic cells from a non-identical donor would have the reciprocal advantage of being naturally free from the disease-causing gene but needing at least some degree of long-term immunosuppression to avoid rejection. In mammals, stem cells with the potential to develop into photoreceptor cells have been obtained from embryonic sources as well as the ciliary body and bone marrow of adults [31, 32]. Several groups have succeeded in surgically implanting these cells into the eyes of animal models affected with a heritable photoreceptor degeneration [33]. In a recent example of such a study, Lamba and coworkers used retinal cells derived from human embryonic stem cells to restore light responses in a mouse model of CRX-associated Leber congenital Amaurosis [34]. A major breakthrough occurred in 2007 when two independent laboratories showed that adult human tissue cells could be reprogrammed to become induced pluripotent stem cells (iPS cells) by transfecting them with four transcription factor genes $[35,36]$. This strategy may prove useful for the creation of patient-specific therapeutic stem cells as well as for the creation of disease-manifesting cell lines for the study of pathophysiologic mechanisms. As a result, iPS cells are already being used to study a number of different diseases ranging from anemia to Parkinson's disease [37, 38].

Another cell-based therapeutic strategy is to use a transplanted cell as a long-term source of a therapeutic protein. For example, in the recent clinical trials of ciliary-derived neurotrophic factor (CNTF), the factor was delivered by implanting a small semi-permeable capsule containing heterologous human cells stably expressing a CNTF transgene [39].

\section{Retinal Prostheses}

The spectacular success of cochlear implants for the treatment of various forms of profound hearing loss have inspired lay people and scientists alike to imagine an analogous treatment for diseases that affect the retina. However, the amount of information gathered and 
transmitted to the brain each second by a healthy retina is easily more than 1000 times that gathered and transmitted by a healthy cochlea. This dramatic bandwidth difference has an anatomic correlate as well. Cochlear implants are linear devices that are threaded into the fluid-filled cochlea through a single small opening while retinal prostheses are planar devices that are intrinsically more difficult to maintain in functional and stable apposition to the cells they are intended to stimulate. However, despite these challenges, great progress has been made with these devices in the past fifteen years. Proof of concept experiments of several different designs have been successful in both animal models and human subjects [40] and at least three companies have announced their intention to have a version of their device ready for use in humans by 2010 [41-43]. The devices under development differ from one another in significant ways. Some stimulate the "subretinal" side of the retina and have the advantage of a simpler spatial relationship between the artificial stimulus and the existing neurological pathways. Other systems stimulate the "epiretinal" surface of the retina and have the advantage that they are a bit safer to implant but the disadvantage that the stimulus has to be created and delivered in a much more sophisticated way to replace the processing that usually occurs in the inner retina. Some systems capture the image with a camera mounted outside the eye and route the information to the retina through a transcutaneous connection while others stimulate self-powered subretinal photodiodes with bright images projected into the eye by special goggles. It seems likely that epiretinal devices connected to extraocular cameras and power sources will be the first to be deployed beyond clinical trials although with some incremental improvements in technology some of the other designs may well supplant them in the future.

\section{Conclusion - Back to the Clinic}

In the past decade, dramatic progress has been made toward the development of a comprehensive set of effective therapies for the wide array of human diseases that affect the photoreceptors. It seems likely that the "new frontiers" of this field will soon be the old frontiers; that is, the eye clinic and the operating room. Now that molecular biology laboratories have identified hundreds of disease-causing genes and given us various highthroughput means for investigating them in individual patients, it will be up to practicing clinicians well-versed in the new molecular lingo to identify the specific patterns and rates of disease that are associated with specific variations in these genes. This will be essential if clinical trials of the promising new therapies summarized in this chapter are to be planned and executed as efficiently as possible. For example, if one unwittingly enrolled a group of patients with severe mutations in a given gene in a "treatment" group while enrolling a group of patients with milder mutations in the "untreated" group, one might erroneously conclude that a treatment didn't work at all when in fact it worked quite well. Making this mistake in the opposite direction might cause one to believe that a treatment worked well when in fact it had no effect. Many photoreceptor diseases are so rare that one will not be able to count on randomization alone to avoid this type of error [8]. What is the most effective way to gather this gene-specific natural history information? One could perform a series of prospective studies and follow patients with known genotypes to determine the rate of progression of various aspects of their disease. This would have the advantage that all of the patients could be examined using identical instrumentation but would have the great 
disadvantage that it would take decades to accomplish for most diseases. An alternative approach would be to identify the disease-causing genotypes of as many patients as possible with as many diseases as possible and to gather, tabulate and analyze the relevant elements of every clinical record that can be obtained from these patients. Very high-resolution natural history data could be generated for the more common alleles of the more common disease genes in a relatively short period of time using this method. This approach would have the added benefit of replacing the decades old message of "there is nothing we can do" with the more hopeful request for a blood sample and a copy of a patient's records for the purpose of trying to cure his or her disease.

\section{Acknowledgements}

The author thanks Audrey Ko, Joseph Bogaard, Jean Andorf, Connie Kuusisto, Arlene Drack, Rob Mullins and Mina Chung for their many helpful suggestions during the preparation of this manuscript. The author's work on photoreceptor degeneration is supported by the Foundation Fighting Blindness, the Carver Endowment for Molecular Ophthalmology, the Grousbeck Family Foundation and Hope for Vision. The author is an Investigator of the Howard Hughes Medical Institute.

\section{References and recommended reading}

Papers of particular interest, published within the period of review, have been highlighted as:

* of special interest

** of outstanding interest

1. Menke MN, et al. Reproducibility of Retinal Thickness Measurements in Healthy Subjects Using Spectralis Optical Coherence Tomography. Am J Ophthalmol. 2008

2*. Daiger SP, Bowne SJ, Sullivan LS. Perspective on genes and mutations causing retinitis pigmentosa. Arch Ophthalmol. 2007; 125(2):151-8. [PubMed: 17296890] [A review article discussing the genetic complexity of retinitis pigmentosa and summarizing the genes that are currently known to cause this disease.]

3. Weleber RG, et al. Phenotypic variation including retinitis pigmentosa, pattern dystrophy, and fundus flavimaculatus in a single family with a deletion of codon 153 or 154 of the peripherin/RDS gene. Arch Ophthalmol. 1993; 111(11):1531-42. [PubMed: 8240110]

4. Klevering BJ, et al. Three families displaying the combination of Stargardt's disease with cone-rod dystrophy or retinitis pigmentosa. Ophthalmology. 2004; 111(3):546-53. [PubMed: 15019334]

5. Hewitt AW, Mackey DA, Craig JE. Myocilin allele-specific glaucoma phenotype database. Hum Mutat. 2008; 29(2):207-11. [PubMed: 17966125]

6. Jacobson SG, et al. Identifying photoreceptors in blind eyes caused by RPE65 mutations: Prerequisite for human gene therapy success. Proc Natl Acad Sci U S A. 2005; 102(17):6177-82. [PubMed: 15837919]

$7 *$. Okou DT, et al. Microarray-based genomic selection for high-throughput resequencing. Nat Methods. 2007; 4(11):907-9. [PubMed: 17934469] [This paper describes a novel microarrarybased method for genomic fragment selection that allows one to focus the power of "next generation" DNA sequencing on specific regions of complex eukaryotic genomes.]

8*. Stone EM. Leber congenital amaurosis - a model for efficient genetic testing of heterogeneous disorders: LXIV Edward Jackson Memorial Lecture. Am J Ophthalmol. 2007; 144(6):791-811. [PubMed: 17964524] [The author describes a multi-platform approach for efficient molecular screening of patients affected with autosomal heterogeneous recessive disorders and demonstrates that a tiered screening strategy is more efficient than an "all genes in all patients" sequencing strategy.] 
9. Stone EM. Finding and interpreting genetic variations that are important to ophthalmologists. Trans Am Ophthalmol Soc. 2003; 101:437-84. [PubMed: 14971589]

10. Berson EL, et al. A randomized trial of vitamin A and vitamin E supplementation for retinitis pigmentosa. Arch Ophthalmol. 1993; 111(6):761-72. [PubMed: 8512476]

11. Li T, et al. Effect of vitamin A supplementation on rhodopsin mutants threonine-17 --> methionine and proline-347 --> serine in transgenic mice and in cell cultures. Proc Natl Acad Sci U S A. 1998; 95(20):11933-8. [PubMed: 9751768]

12**. Radu RA, et al. Accelerated accumulation of lipofuscin pigments in the RPE of a mouse model for ABCA4-mediated retinal dystrophies following Vitamin A supplementation. Invest Ophthalmol Vis Sci. 2008; 49(9):3821-9. [PubMed: 18515570] [This study demonstrates a detrimental effect of dietary vitamin A supplementation in abca4 deficient mice suggesting that vitamin A supplementation should be avoided in patients with retinal dystrophies caused by mutations in ABCA4.]

13. A randomized, placebo-controlled, clinical trial of high-dose supplementation with vitamins $\mathrm{C}$ and $\mathrm{E}$, beta carotene, and zinc for age-related macular degeneration and vision loss: AREDS report no. 8. Arch Ophthalmol. 2001; 119(10):1417-36. [PubMed: 11594942]

14. Hao W, et al. Evidence for two apoptotic pathways in light-induced retinal degeneration. Nat Genet. 2002; 32(2):254-60. [PubMed: 12219089]

15. Cideciyan AV, et al. In vivo dynamics of retinal injury and repair in the rhodopsin mutant dog model of human retinitis pigmentosa. Proc Natl Acad Sci U S A. 2005; 102(14):5233-8. [PubMed: 15784735]

16. Mata NL, Weng J, Travis GH. Biosynthesis of a major lipofuscin fluorophore in mice and humans with ABCR-mediated retinal and macular degeneration. Proc Natl Acad Sci U S A. 2000; 97(13): 7154-9. [PubMed: 10852960]

17. Radu RA, et al. Reductions in serum vitamin A arrest accumulation of toxic retinal fluorophores: a potential therapy for treatment of lipofuscin-based retinal diseases. Invest Ophthalmol Vis Sci. 2005; 46(12):4393-401. [PubMed: 16303925]

18. Anderson B Jr. Ocular effects of changes in oxygen and carbon dioxide tension. Trans Am Ophthalmol Soc. 1968; 66:423-74. [PubMed: 5720847]

19. Komeima K, et al. Antioxidants reduce cone cell death in a model of retinitis pigmentosa. Proc Natl Acad Sci U S A. 2006; 103(30):11300-5. [PubMed: 16849425]

20*. Phillips MJ, et al. Tauroursodeoxycholic acid preservation of photoreceptor structure and function in the rd10 mouse through postnatal day 30. Invest Ophthalmol Vis Sci. 2008; 49(5):2148-55. [PubMed: 18436848] [This study showed that tauroursodeoxycholic acid (TUDCA) was able to preserve some photoreceptor function in the rd10 mouse model of retinitis pigmentosa suggesting a potential role for this agent in patients with retinitis pigmentosa.]

21. Delyfer MN, et al. Inherited retinal degenerations: therapeutic prospects. Biol Cell. 2004; 96(4): 261-9. [PubMed: 15145530]

22. Rhee KD, et al. Molecular and cellular alterations induced by sustained expression of ciliary neurotrophic factor in a mouse model of retinitis pigmentosa. Invest Ophthalmol Vis Sci. 2007; 48(3):1389-400. [PubMed: 17325188]

23. Cai X, Conley S, Naash M. Nanoparticle applications in ocular gene therapy. Vision Res. 2008; 48(3):319-24. [PubMed: 17825344]

24. Acland GM, et al. Gene therapy restores vision in a canine model of childhood blindness. Nat Genet. 2001; 28(1):92-5. [PubMed: 11326284]

25. Maguire AM, et al. Safety and efficacy of gene transfer for Leber's congenital amaurosis. N Engl J Med. 2008; 358(21):2240-8. [PubMed: 18441370]

26. Bainbridge JW, et al. Effect of gene therapy on visual function in Leber's congenital amaurosis. N Engl J Med. 2008; 358(21):2231-9. [PubMed: 18441371]

$27 * *$. Hauswirth W, et al. Phase I Trial of Leber Congenital Amaurosis due to RPE65 Mutations by Ocular Subretinal Injection of Adeno-Associated Virus Gene Vector: Short-Term Results. Hum Gene Ther. 2008 [References 25-27 each summarize the results of one of the first clinical trials of gene replacement therapy for a human photoreceptor disease. In each study, subretinal 
delivery of a recombinant adeno-associated virus bearing a functional copy of RPE65 resulted in some improvement of visual function without any serious adverse effects.]

28. Gorbatyuk M, et al. Preservation of photoreceptor morphology and function in $\mathrm{P} 23 \mathrm{H}$ rats using an allele independent ribozyme. Exp Eye Res. 2007; 84(1):44-52. [PubMed: 17083931]

29. Buch PK, et al. In contrast to AAV-mediated Cntf expression, AAV-mediated Gdnf expression enhances gene replacement therapy in rodent models of retinal degeneration. Mol Ther. 2006; 14(5):700-9. [PubMed: 16872907]

30. Stieger K, et al. Oral administration of doxycycline allows tight control of transgene expression: a key step towards gene therapy of retinal diseases. Gene Ther. 2007; 14(23):1668-73. [PubMed: 17914405]

31. Gong L, et al. Differentiation of rat mesenchymal stem cells transplanted into the subretinal space of sodium iodate-injected rats. Clin Experiment Ophthalmol. 2008; 36(7):666-71. [PubMed: 18983552]

32. Jomary C, Jones SE. Induction of functional photoreceptor phenotype by exogenous Crx expression in mouse retinal stem cells. Invest Ophthalmol Vis Sci. 2008; 49(1):429-37. [PubMed: 18172122]

33. Wang S, et al. Long-term vision rescue by human neural progenitors in a rat model of photoreceptor degeneration. Invest Ophthalmol Vis Sci. 2008; 49(7):3201-6. [PubMed: 18579765]

34. Lamba DA, Gust J, Reh TA. Transplantation of human embryonic stem cell-derived photoreceptors restores some visual function in crx-deficient mice. Cell Stem Cell. 2009; 4(1):739. [PubMed: 19128794]

35. Takahashi K, et al. Induction of pluripotent stem cells from adult human fibroblasts by defined factors. Cell. 2007; 131(5):861-72. [PubMed: 18035408]

36*. Yu J, et al. Induced pluripotent stem cell lines derived from human somatic cells. Science. 2007; 318(5858):1917-20. [PubMed: 18029452] [In references 35 and 36 the authors demonstrate that human somatic cells can be reprogrammed into pluripotent stem cells by transduction with a combination of four genes.]

37*. Amabile G, Meissner A. Induced pluripotent stem cells: current progress and potential for regenerative medicine. Trends Mol Med. 2009 [A review article that compares studies of embryonic stem (ES) cells to those of induced pluripotent stem (iPS) cells.]

38*. Hochedlinger K, Plath K. Epigenetic reprogramming and induced pluripotency. Development. 2009; 136(4):509-23. [PubMed: 19168672] [This review article provides a broad overview of the induction of pluripotency in somatic cells and discusses both advances and limitations in the cell reprogramming field.]

39. Sieving PA, et al. Ciliary neurotrophic factor (CNTF) for human retinal degeneration: phase I trial of CNTF delivered by encapsulated cell intraocular implants. Proc Natl Acad Sci U S A. 2006; 103(10):3896-901. [PubMed: 16505355]

40**. Dowling J. Current and future prospects for optoelectronic retinal prostheses. Eye. 2008 [This review article summarizes the different approaches to the development of a practical retinal prosthesis for the treatment of degenerative retinal diseases.]

41. Yanai D, et al. Visual performance using a retinal prosthesis in three subjects with retinitis pigmentosa. Am J Ophthalmol. 2007; 143(5):820-827. [PubMed: 17362868]

42. Hornig R, et al. A method and technical equipment for an acute human trial to evaluate retinal implant technology. J Neural Eng. 2005; 2(1):S129-34. [PubMed: 15876648]

43. Besch D, et al. Extraocular surgery for implantation of an active subretinal visual prosthesis with external connections: feasibility and outcome in seven patients. Br J Ophthalmol. 2008; 92(10): 1361-8. [PubMed: 18662916] 



Figure.

Panel A - A montage of fundus photographs from the right eye of a 19 year old patient affected with autosomal dominant retinitis pigmentosa caused by a heterozygous Gly114Asp variation in the rhodopsin gene. The "bone-spicule-like" pigmentation that gives the condition its name is most prominent anterior to the major vascular arcades.

Panel B - Higher magnification view of the macula of the same eye shown in panel A. The retinal arterioles (arrowheads) have vasoconstricted in response to the high oxygen tension in the retina and are so narrow that they are almost invisible while the venules remain near normal in caliber. Retinal edema is present in the macula and a single large cyst is present in the fovea (arrow). 
Table 1

Six Classes of Therapy for Inherited Photoreceptor Degenerations

Genetic testing

Environmental modification

Nutritional supplementation

Toxin avoidance

Drugs

Small molecule drugs (e.g. vitamins)

Large molecule drugs (e.g. growth factors)

Gene therapy

Gene replacement

Mutant allele suppression

Large molecule drug delivery

Cell therapy

Replacement of differentiated cell function

Replacement of supportive cell function

Large molecule drug delivery

Retinal prostheses 